\title{
Correlation between Clinical Factors and Prognosis in Newly Diagnosed Multiple Myeloma
}

\author{
Xiao-Qing Zhao, Shu-Ying Zhao, Wen-Xin Chen, Xiao-Wei Liu, Huai-Xiu Yan and Yong-Ji Lou \\ Department of Hematology, The Third Affiliated Hospital of Inner Mongolia Medical University, Baotou, China
}

\begin{abstract}
Objective: To explore the correlation between clinical factors and the prognosis of multiple myeloma (MM).

Study Design: A cohort study.

Place and Duration of Study: At the Third Affiliated Hospital of Inner Mongolia Medical University, Mongolia from January 2014 to December 2018.

Methodology: The clinical data of 81 patients with newly diagnosed MM were collected retrospectively. The correlation of prognosis with immunophenotype and (FISH) Fluorescence in situ hybridization was subjected to univariate and multivariate analyses. Overall and progression-free survival was determined.

Results: The overall survival (OS) and progression-free survival (PFS) of patients with CD200+, CD81+, and CD27- were significantly shortened. CD200+ and CD27- were independent prognostic factors for OS and PFS in MM patients. The prognosis-related abnormal genes were analyzed, and univariate analysis revealed that OS and PFS were significantly shortened in patients with RB-1 deletion, CDKN2C deletion, and IGH rearrangement. CDKN2C deletion was an independent prognostic factor for OS and PFS in MM patients.

Conclusion: CDKN2C deletion is an independent prognostic factor of MM. CD200+ is an independent risk factor for poor prognosis of MM.
\end{abstract}

Key Words: Multiple myeloma, Prognostic analysis, Fluorescence in situ hybridization, Immunity, Immunophenotype.

How to cite this article: Zhao XQ, Zhao SY, Chen WX, Liu XW, Yan HX, Lou YJ. Correlation between Clinical Factors and Prognosis in Newly Diagnosed Multiple Myeloma. J Coll Physicians Surg Pak 2020; 30(06):601-605 https://doi.org/10.29271/jcpsp.2020.06.601.

\section{INTRODUCTION}

Multiple myeloma (MM) is a clonal B-cell disease, and its pathogenesis is due to the uncontrolled and destructive growth of abnormal plasma cells that accumulate in the bone marrow. ${ }^{1,2}$ Approximately 20,000 new patients are diagnosed in the United States and Europe each year. There is no cure for $\mathrm{MM}^{3},{ }^{3,4}$ therefore, one needs to continually evaluate the prognosis of MM patients to understand the progress of the disease. This can help in providing accurate and holistic treatment to help prolong the survival of the patients.

Various studies have reported inconsistent prognostic factors of MM. ${ }^{5}$ Prognosis was evaluated through the Durie-Salmon ( $D$ S) staging system (based on serum or urinary protein content, hemoglobin, blood calcium, serum creatinine, severity of bone destruction, and renal function index) and the International Staging System (ISS) (based on $\beta 2$-microglobulin and serum albumin levels).

Correspondence to: Wen-Xin Chen, Department of Hematology, The Third Affiliated Hospital of Inner Mongolia Medical University, No.20 Shaoxian Road, Kundulun

District, Baotou, 014010, China

E-mail: chen_wenxin77@163.com

Received: July 29, 2019; Revised: June 04, 2020;

Accepted: June 18, 2020

DOI: https://doi.org/10.29271/jcpsp.2020.06.601
In this era of new diagnostic methods, studies have revealed that cytogenetics can provide prognostic factors independent of ISS. Therefore, ISS was revised to become the Revised International Staging System (R-ISS) by combining ISS staging, cytogenetics, and lactate dehydrogenase. This new staging can better determine an outcome, and some cytogenetic abnormalities have been incorporated into prognostic stratification. ${ }^{6}$ In addition, flow cytometry provides an important basis for the diagnosis and immunophenotypic analysis of MM and improves its diagnostic level, playing a guiding role in the treatment and prognosis of MM patients. $^{7}$

To better understand the prognostic factors of MM patients, the present study analyzed the clinical data of 81 newly diagnosed MM patients from our hospital. The prognostic factors of flow cytometric immunophenotype and fluorescence in situ hybridization (FISH) were evaluated for the diagnosis, treatment, and prognosis of MM patients.

\section{METHODOLOGY}

The clinical data (general clinical data, laboratory tests, and prognostic factor analyses) were retrospectively collected from 81 patients with MM diagnosed between January 2014 to December 2018 at Medical College of Inner Mongolia Medical University Mongolia. The diagnoses of the selected patients were made according to the criteria outlined by the National Comprehensive Cancer Network of the United States and the International Myeloma Working Group. ${ }^{8}$ Solitary plasmacytoma and primary plasmacyticleukemia were excluded. 
Table I: The relationship between abnormal immunophenotype and OS, PFS.

\begin{tabular}{|c|c|c|c|c|c|c|c|}
\hline \multirow{2}{*}{ Immunophenotype } & \multirow{2}{*}{$\mathbf{N}$} & \multicolumn{3}{|c|}{ OS } & \multicolumn{3}{|c|}{ PFS } \\
\hline & & Median (M) & Mean value (M) & p-value & Median (M) & Mean value (M) & p-value \\
\hline CD200 & & & & 0.005 & & & 0.002 \\
\hline Positive & 17 & 42 & 29.84 & & 32 & 25.65 & \\
\hline Negative & 30 & 43 & 45.37 & & 38 & 40.5 & \\
\hline CD27 & & & & $<0.001$ & & & $<0.001$ \\
\hline Positive & 15 & 18 & 19.64 & & 18 & 19.64 & \\
\hline Negative & 32 & 42 & 44.35 & & 38 & 38.81 & \\
\hline CD81 & & & & 0.012 & & & 0.001 \\
\hline Positive & 13 & 32 & 29.71 & & 32 & 25.69 & \\
\hline Negative & 34 & 42 & 44.47 & & 38 & 42.55 & \\
\hline
\end{tabular}

Table II: The relationship between FISH detection of abnormal genes and OS, PFS

\begin{tabular}{|c|c|c|c|c|c|c|c|}
\hline \multirow{2}{*}{ Abnormal gene } & \multirow{2}{*}{$\mathbf{N}$} & \multicolumn{3}{|c|}{ OS } & \multicolumn{3}{|c|}{ PFS } \\
\hline & & Median (M) & Mean value (M) & p-value & Median (M) & Mean value (M) & p-value \\
\hline RB-1 deletion & & & & 0.027 & & & 0.033 \\
\hline Positive & 19 & 27 & 28.81 & & 27 & 26.04 & \\
\hline Negative & 22 & 43 & 37 & & 38 & 34.14 & \\
\hline CDKN2C deletion & & & & $<0.001$ & & & $<0.001$ \\
\hline Positive & 14 & 21 & 23.54 & & 21 & 22.26 & \\
\hline Negative & 27 & 43 & 43.6 & & 38 & 38 & \\
\hline IGH re-arrangement & & & & 0.021 & & & 0.001 \\
\hline Positive & 9 & 21 & 22.39 & & 21 & 20.11 & \\
\hline Negative & 32 & 42 & 35.57 & & 38 & 32.37 & \\
\hline
\end{tabular}

Cytogenetics, including FISH, routine chromosomes, and flow cytometric immunophenotypes of newly diagnosed MM patients, were collected, and the findings were sent to the Hematological Hospital of Medical Sciences (Xiehe, Huamei, China). Study subjects were staged by the D-S staging system, ISS, and R-ISS. ${ }^{6}$ Follow-up and data collection of MM patients from the time of definitive diagnosis until September 2018, were gathered by case review, telephone calls, and outpatient follow-up.

All data were analysed using the statistical software SPSS 21.0. Progression-free survival (PFS) was defined as the duration from the start of treatment to the progression of the disease or patient death. The events analysed for PFS included disease progression, relapse, and patient death. Overall survival (OS) analysis was only used where there was a patient death; it was defined as the duration from diagnosis to the patient's death. Qualitative variables were expressed as frequencies and percentages, and quantitative variables were expressed as medians and range. KaplanMeier method was used for survival analysis, and the survival rate curve was drawn. Log-rank test was used for univariate prognostic analysis. Multivariate prognostic analysis was conducted using the Cox hazard regression model. $\mathrm{P}<0.05$ was considered statistically significant.

\section{RESULTS}

Of the $81 \mathrm{MM}$ patients, $45(55.56 \%)$ patients were males, and $36(44.44 \%)$ were females, with a male-to-female ratio of 1.24:1. The median age was 71 years (44 to 87 years old). Among these patients, 40 patients (49.4\%) were younger than 70 years, and $41(50.6 \%)$ were older.

The proportions of immunoglobulin $\mathrm{G}$ type and immunoglobulin A type were relatively high $32.1 \%$ (26 patients) and 30.9\% (25 patients), respectively. Eighteen patients (22.2\%) were light-chain type, seven patients (8.6\%) were immunoglobulin D type, and five patients $(6.2 \%)$ were the non-secretory type.

D-S staging was performed on 81 MM patients: four patients (4.9\%) were at stage I-A; 21 patients $(25.9 \%)$ were at stage II$A$; three patients (3.7\%) were at stage II-B; 39 patients (48.1\%) were at stage III-A; and 14 patients (17.3\%) were at stage III-B. The majority of MM patients were at stage III and had normal renal function, and no MM patients were found at stage I-B. Of the 81 MM patients, the majority (37 [45.7\%]) were at ISS stage III; 26 patients (32.1\%) were at stage II, and 18 patients $(22.2 \%)$ were at stage I. Of the 81 MM patients, 44 MM patients could be staged using the R-ISS staging system. Among these patients, 6 (14.6\%) were at stage I, 11 (26.8\%) were at stage II, and 27 (65.9\%) were at stage III. The proportion of patients at stage III in the R-ISS staging system was higher than that in the ISS staging system. 


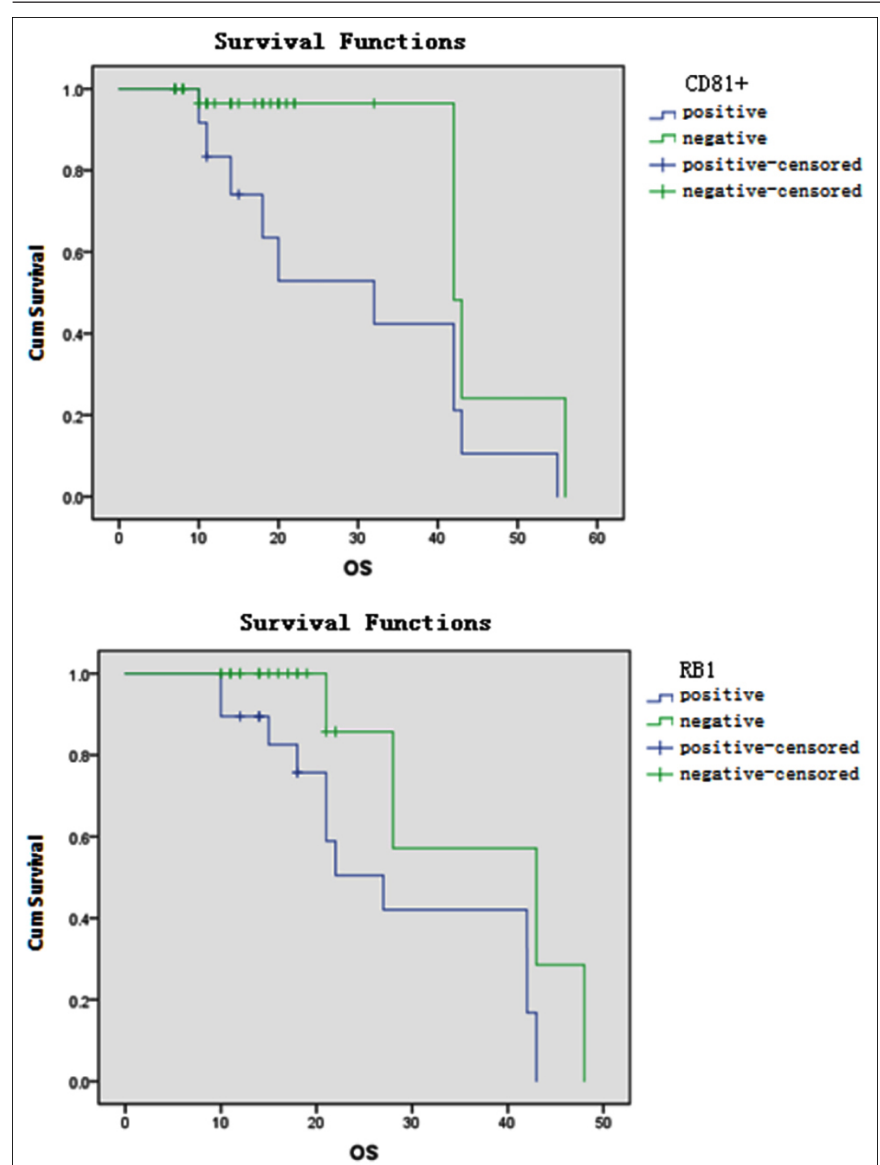

Figure 1: Effects of CD81+ and RB-1 deletion on OS in MM patients.

Flow cytometry was performed in 47 of the 81 newly diagnosed patients, and their abnormal detection rate was $100 \%$. Phenotypes and proportions: CD38+ was detected in 47 patients (100\%); CD138 + was detected in 44 patients (94.7\%); CD56 + was detected in 24 patients (51.1\%); CD117+ was detected in 23 patients (48.9\%); CD200+ was detected in 17 patients (36.2\%); CD27+ was detected in 14 patients $(29.8 \%)$; CD20 + was detected in 13 patients (27.7\%); CD81+ was detected in 13 patients $(27.7 \%)$; $\mathrm{CD} 10+$ was detected in four patients $(8.5 \%)$; CD14+ was detected in three patients $(6.4 \%)$; CD16+ was detected in three patients (6.4\%); and CD33+ was detected in two patients $(4.3 \%)$.

Of the 81 newly diagnosed MM patients, routine chromosome detection was performed in 41 patients. In four patients, cleavage was not found. In the detection reports of 37 patients, normal karyotype was detected in 28 patients $(75.7 \%)$, and abnormal karyotype was detected in nine patients (24.3\%).
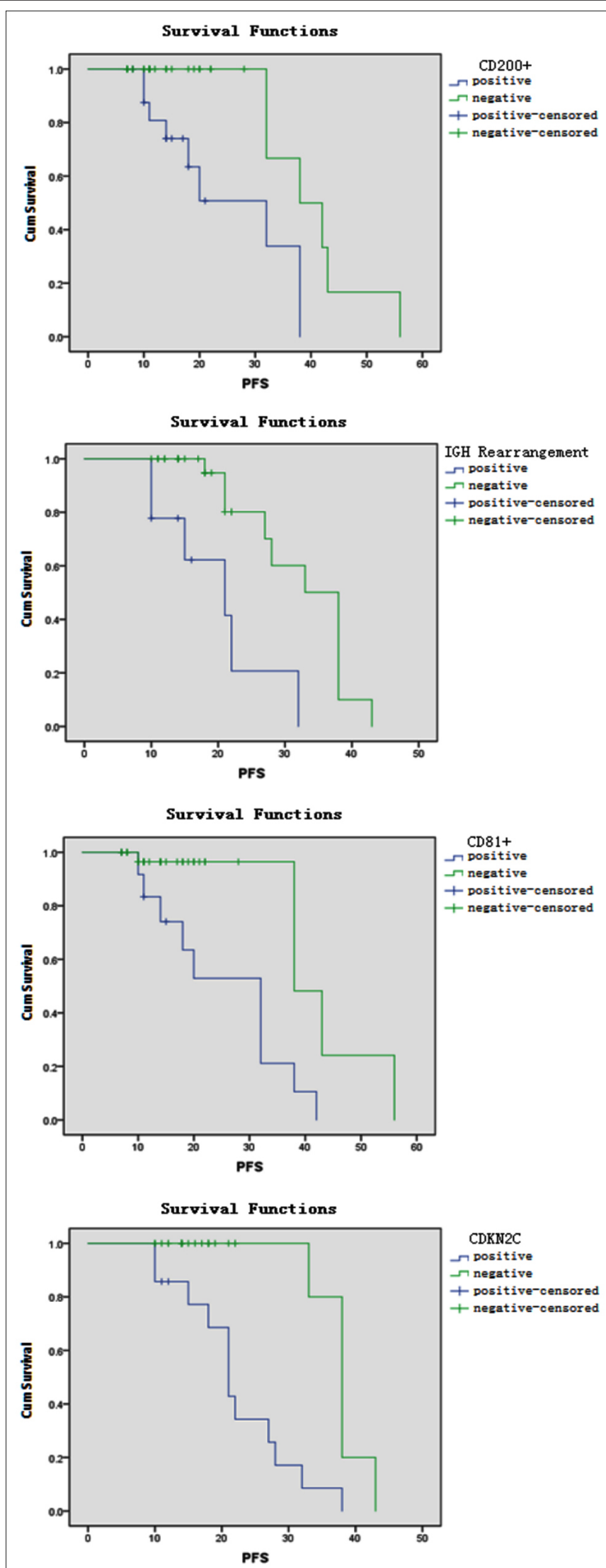

Figure 2: Effects of CD200+, CD81+, IGH rearrangement and CDKN2C deletion on PFS in MM patients.

FISH was performed in 41 of the 81 patients in the study, 
and abnormalities were detected in 30 patients (73.2\%). The abnormal genes and detection rate were IGH/FGFR3 fusion gene, $7.3 \%$ (three patients); CCND1/IGH fusion gene, $14.6 \%$ (six patients); RB-1 deletion, 46.3\% (19 patients); CKS1B amplification, 41.4\% (17 patients); TP53 deletion, 29.3\% (12 patients); CDKN2C deletion, 34.1\% (14 patients); and IGH rearrangement, $21.9 \%$ (nine patients).

Forty-seven patients with complete flow cytometry were analysed for prognostic factors. The results revealed that the groups with CD200, CD27, and CD81 had similar lengths of OS ( $p=0.005,<0.001$, and 0.012 , respectively) and PFS ( $p$ $=0.002,<0.001$, and 0.001 , respectively); the OS and PFS were significantly shortened in patients with CD200+, CD27-, and CD81 (Table I, Figures 1 and 2).

Forty-one patients with complete FISH detection were analysed for prognostic factors. The results showed that the groups with RB-1 deletion, CDKN2C deletion, and IGH rearrangement had similar OS survival times ( $p=0.027$, $<0.001$, and 0.021 , respectively) and PFS ( $p=0.033$, $<0.001$, and 0.001 , respectively); the OS and PFS were significantly shortened in patients with RB-1 deletion, CDKN2C deletion, and IGH rearrangement (Table II, Figures 1 and 2).

OS and PFS indexes from the univariate analysis of 47 patients with complete flow cytometry, including CD200, CD81, and CD27, were analysed using the Cox proportional hazards regression model. The results showed that CD200+ and CD27- were independent prognostic factors for OS ( $p=$ 0.033 and 0.009 , respectively), and CD200+, CD27-, and CD81 were independent prognostic factors for PFS ( $p=$ $0.005,0.035$, and 0.033).

Indices associated with OS and PFS from the univariate analysis of 41 patients with complete FISH detection, including RB-1 deletion, CDKN2C deletion, and IGH rearrangement, were analysed using the Cox proportional hazards regression model. The results revealed that CDKN2C deletion was an independent prognostic factor for OS $(p=0.023)$ and PFS ( $p$ $=0.026)$ in MM patients.

\section{DISCUSSION}

MM has complex pathogenesis and prognostic factors, including the effects of changes in endocrine, vascular, genetic, and metabolic factors. However, cellular immune markers, heredity, and gene status are essential in $\mathrm{MM}^{9}$

CD200 is a transmembrane glycoprotein and plays a role in immune regulation and tolerance. It is expressed in many cell types and highly expressed in B-cell malignant tumors and acute myeloid leukemia. ${ }^{10}$ Oltenau et al. confirmed that CD200 was also highly expressed in human myeloma samples. ${ }^{11}$ Moreaux et al. studied the correlation between the mRNA expression of CD200 and the decrease in survival time (14 or 24 months) in MM patients; ${ }^{12}$ CD200 expression was a poor prognostic factor for MM.

Wang et al. showed that the clinical characteristics of MM patients with CD27- and CD27+ were not significantly related to genetic abnormalities. ${ }^{13}$ The results suggest that the prognosis of CD27- patients is less favorable than that of CD27+ patients. However, the number of cases in the present study was relatively small; therefore, the prognostic evaluation of CD27 needs further study. CD56, CD20, and CD117 are expressed in abnormal plasma cells, and other studies have analysed their prognostic value, indicating that CD56+ and CD117+ were useful prognostic markers, while CD20 revealed disease progression and was an indicator of poor prognosis. ${ }^{14}$ In the present study, univariate analysis of CD56, CD20, and CD117 was performed. The results need to be verified by further research. However, a study reported that the prognosis of newly diagnosed MM patients with CD19+ CD81+ was poorer than that of newly diagnosed MM patients with CD19- CD81-. ${ }^{15}$ In the present study, the detection rates of CD38+ and CD138+ were $100 \%$ and $94.7 \%$, respectively, which aligns with the results reported in the literature.

When an autosomal examination is performed in MM patients, because FISH detection is not restricted by other factors in the separation of plasma cells, ${ }^{16}$ the abnormal detection rate was $73.1 \%$ in the present study, which is similar to the results by $\mathrm{Wu} .{ }^{17} \mathrm{RB}-1$ gene deletion, caused by complete or partial deletion of chromosome 13 , is a common chromosomal abnormality in MM, can be detected in $40 \%$ of MM patients, ${ }^{18}$ and is an important prognostic factor for early detection. A study revealed that it was not an independent prognostic factor, and its poor prognosis was primarily correlated to the combination of $17 \mathrm{P}-, \mathrm{t}(4 ; 16)$ and $\mathrm{t}(14 ; 16)$ and other unfavourable prognostic factors. ${ }^{19}$ The translocation or deletion affecting $14 q 32$ induces IGH rearrangement. For chromosome 14q32 translocation, the most common changes were $t(11 ; 14)$ (q13; q32), t $(4 ; 14)$ ( $16 ; q 32)$, and t $(14 ; 16)(q 32 ; q 32)$ translocations, and the fusion genes formed were CCND1/IGH, IGH/FGFR3, and IGH/MAF. In the present study, the IGH/MAF fusion gene was not detected in any MM patients, and the remaining abnormal genes were analysed by univariate analysis. The results demonstrated that RB-1 gene deletion, CDKN2C gene deletion, and IGH rearrangement were factors predicting a poor prognosis. Multivariate analysis revealed that: CDKN2C gene deletion was an independent risk factor for an unfavorable prognosis of MM. There are few Chinese studies on CDKN2C gene deletion caused by a 1p32 deletion, and research is still needed.

\section{CONCLUSION}

CD200+ is an independent risk factor for poor prognosis of MM. CDKN2C deletion is an independent prognostic factor of MM patients. For confirmation of these findings and further analysis of prognostic factors of MM, a larger sample size and multi-centre data analysis are needed.

\section{ETHICAL APPROVAL:}


This study was conducted with approval from the Ethics Committee of the Third Affiliated Hospital of Inner Mongolia Medical University. This study was conducted in accordance with the Declaration of Helsinki. Written informed consents were obtained from all participants.

\section{PATIENTS' CONSENT:}

All participants signed a document of informed consent.

\section{AUTHORS' CONTRIBUTION:}

WXC, XWL, XQZ: Acquired data.

WXC, SYZ, XQZ: Drafted the manuscript.

SYZ, HXY, YJL: Contributed substantially to its revision.

All authors read and approved the final manuscript.

\section{CONFLICT OF INTEREST:}

The authors declare that they have no competing interests.

\section{FUNDING:}

Baotou Science and Technology Project (No. 2018C2007-3-18).

\section{REFERENCES}

1. Fairfield H, Falank C, Avery L, Reagan MR. Multiple myeloma in the marrow: Pathogenesis and treatment. Ann N Y Acad Sci 2016; 1364:32-41.

2. Sultan S, Irfan SM, Parveen S, Ali H, Basharat M. Multiple Myeloma: A retrospective analysis of 61 patients from a teryiary care center. Asian Pac J cancer Prev 2016; 17(4): 1833-4.

3. Raab MS, Podar K, Breitkreutz I, Richardson PG, Anderson KC. Multiple myeloma. Lancet 2009; 374:324-39.

4. Teitelbaum A, Ba-Mancini A, Huang H, Henk HJ. Healthcare costs and resource utilization, including patient burden, associated with novel-agent-based treatment versus other therapies for multiple myeloma: Findings using real-world claims data. Oncologist 2013; 18(1):37-45.

5. Hose $D$, Rème $T$, Hielscher $T$, Moreaux J, Messner $\mathrm{T}$, Seckinger $\mathrm{A}$, et al. Proliferation is a central independent prognostic factor and target for personalised and risk-adapted treatment in multiple myeloma. Haematologica 2011; 96(1):87-94.

6. Palumbo A, Avet-Loiseau H, Oliva S. Revised international staging system for multiple myeloma: A report from international myeloma working group. J Clin Oncol 2015; 33(26):2863-9.

7. Alaterre E, Raimbault S, Garcia JM, Rème $T$, Requirand G, Klein $B$, et al. Automated and simplified identification of normal and abnormal plasma cells in multiple myeloma by flow cytometry. Cytometry B Clin Cytom 2018; 94(3):484-92.

8. Rajkumar SV, Dimopoulos MA, Palumbo A, Blade J, Merlini G, Mateos MV, et al. International myeloma working group updated criteria for the diagnosis of multiple myeloma. Lancet Oncol 2014; 15(12):e438-e48.

9. Fairfield H, Falank C, Avery L, Reagan MR. Multiple myeloma in the marrow: Pathogenesis and treatments. Ann NY Acad Sci 2016; 1364(1):32-51.

10. Conticello C, Giuffrida R, Parrinello N, Buccheri S, Adamo L, Sciuto MR, et a1. CD200 expression in patients with multiple myeloma: Another piece of the puzzle. Leuk Res 2013; 37(12):1616-21.

11. Olteanu $H$, Harrington AM, Hari P, Kroft SH. CD200 expression in plasma cell myeloma. Br J Haematol 2011; 153(3): 408-11.

12. Moreaux J, Hose D, Reme T, Jourdan E, Hundemer M, Legouffe $\mathrm{E}$, et al. CD200 is a new prognostic factor in multiple myeloma. Blood 2006; 108(13):4194-7.

13. Wang H, Liu L, Liu F, Chen LJ, Qu XY, Li JY, et al. Diagnostic value of cd27 antigen in patients with multiple myeloma. Zhongguo Shi Yan Xue Ye Xue Za Zhi 2017; 25(4): 1069-73.

14. Chang ZW, Zhu HF, Feng MJ, Su XL, Hou LP, Dong Y, et al. Immunophenotypic characteristics and clinical significance of 160 patients with multiple myeloma. Prog Modern Biomed 2016; 16:5179-83, 5148.

15. Paiva B, Puig N, Cedena MT, Ruiz Y, Rapado I, MartinezLopez J, et al. Differentiation stage of myeloma plasma cells: Biological and clinical significance. Leukemia 2017; 31(2):382-92.

16. Kapoor P, Fonseca R, Rajkumar SV, Sinha S, Gertz MA, Stewart AK, et al. Evidence for cytogenetic and fluorescence in situ hybridization risk stratification of newly diagnosed multiple myeloma in the era of novel therapies. Mayo Clin Proc 2010; 85(6):432-7.

17. Wu H, Zhang $\mathrm{H}, \mathrm{He} H g Y$. Cytogenetic abnormalities and prognosis of 432 patients with multiple myeloma. Zhonghua Xue Ye Xue Za zhi 2017; 38:739-43.

18. Walker BA, Leone PE, Chiecchio L, Dickens NJ, Jenner MW, Boyd KD, et al. A compendium of myeloma-associated chromosomal copy number abnormalities and their prognostic value. Blood 2010; 116(15):e56-65.

19. Loiseau AH, Attal M, Moreau P, Charbonnel C, Garban F, Hulin $C$, et al. Genetic abnormalities and survival in multiple myeloma: The experience of the intergroupe francophone du myelome. Blood 2007; 109(8):3489-94. 